\title{
A revision of Foetidia (Lecythidaceae subfamily Foetidioideae)
}

\author{
Ghillean T. Prance
}

School of Biological Sciences, University of Reading, Whiteknights, Reading, RG6 6AS, UK; e-mail: gtolmiep@aol.com

\begin{abstract}
Seventeen species of Foetidia are recognized; one from East Africa, two from the Mascarene Islands and the remaining fourteen from Madagascar. A key to all the species is provided for the first time.
\end{abstract}

Key Words: Foetidia, Lecythidaceae, Madagascar, Mascarenes.

As part of an ongoing study of the phylogenetic relationships of the genera of Lecythidaceae, a revision of the little-studied genus Foetidia Comm. ex Lam. is presented here. Foetidia is included in the Lecythidaceae here as the subfamily Foetidioideae (see also Tsou, 1994), but there are also arguments for treating it as a family sister to the Barringtoniaceae (= Lecythidaceae subfam. Planchonioideae) which in turn is sister to the Lecythidaceae (Mori et al., 2007). In his monograph of the family, Knuth (1939) recognized five species of Foetidia, one of which is excluded here. He placed the genus in a separate tribe, Foetidieae in his family Barringtoniaceae. Bosser (1988) described ten new species from Madagascar but did not produce a key, and Verdcourt (1985) described the first known species of this genus from Africa. These species have not previously been brought together into a single publication and there is no key for their identification except the outdated one of Knuth (1939) in which only four species of Foetidia were treated. This publication treats together the African, Madagascan, and Mascarene species for the first time. For species with many specimens I have cited just one for each Province of Madagascar. Data about many more specimens of some species can be found in the Tropicos database (http://mobot. mobot.org/W3T/search/vast/html).

Foetidia Comm. ex Lam., Encycl. 2 (2): 457. 1788. Type: F. mauritiana Lam.

Trees or shrubs. Leaves alternate but grouped towards the end of branches, usually glabrous, often asymmetric, stipules absent. Flowers usually solitary in axils or rarely in axillary or terminal few-flowered fascicles; pedicels bearing two opposite bracteoles. Receptacle obpyramidal to conical; perianth of 4 (rarely 3 ) persistent tepals, free or slightly fused at base; stamens many, multiseriate; anthers oblong with longitudinal slits, basifixed, introrse; ovary inferior, 2 or 4 locular, with many ovules in each loculus (5-30), placentation axile; ovules anatropous but some curved and somewhat campylotropous; style filiform, (2-)4(5) short-branched; disc epigynous, bearing style at center. Fruit turbinate with persistent tepals, indehiscent, 1-4 locular, with one or rarely two exarillate seeds developing in each loculus.

Distribution.-Mainly Madagascar and the Mascarene Islands with one species in Tanzania.

\section{Key to species of Foetidia}

1. Flowers in axillary and terminal clusters of $2-6$.

2. Bracteoles at base of pedicels; tepals $3-4 \times 2-2.5 \mathrm{~mm}$.

2. Bracteoles on pedicels at base of receptacle; tepals $10-14 \times 4-6 \mathrm{~mm}$.

3. Leaves sessile, oblong, the apex usually acute, rarely rounded..... F. parviflora F. obliqua 
3. Leaves petiolate, broadly elliptic to obovate, the apex retuse or rounded.

4. Leaves $2.5-5.5 \times 1.3-3.2 \mathrm{~cm}$, drying gray nitid; midrib impressed above; petioles $1-5 \mathrm{~mm}$ long....F. delphinensis

4. Leaves $2.5-9.5 \times 1.4-6 \mathrm{~cm}$, drying dull brown; midrib plane above; petioles $5-14 \mathrm{~mm}$ long...........F. retusa 1. Flowers solitary in axils of leaves.

5. Leaves petiolate.

6. Midrib slightly impressed on upper leaf surface.

7. Fruiting tepals $2.5-3.5 \times 1.2-1.5 \mathrm{~cm}$; pedicels $3-5 \mathrm{~cm}$; leaves $6-13 \mathrm{~cm}$ long............... sambiranensis

7. Fruiting tepals $4-12 \times 2-8 \mathrm{~mm}$; pedicels $0.8-1.5 \mathrm{~cm}$; leaves $1.3-6 \mathrm{~cm}$ long.

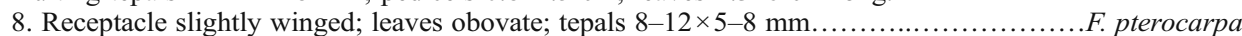

8. Receptacle not winged; leaves cuneiform; tepals $4-7 \times 2-2.5 \mathrm{~mm} \ldots \ldots \ldots \ldots \ldots \ldots \ldots \ldots \ldots \ldots \ldots \ldots \ldots$ cuneata

6. Midrib plane or prominulous on leaf upper surface.

9. Leaves $9-19 \times 3.7-7 \mathrm{~cm}$, thinly papyraceous, the apex shortly acuminate; tepals not constricted at

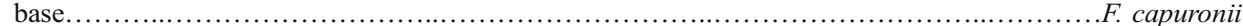

9. Leaves $1.5-6 \times 1-4.5 \mathrm{~cm}$, coriaceous or chartaceous, the apex rounded or retuse; tepals constricted at base (unguiculate).....

5. Leaves sessile or subsessile.

10. Midrib impressed on upper leaf surface.

11. Pedicels 4-6.5 cm long; tepals $3.5-5.5 \times 2-4 \mathrm{~cm}$, deeply constricted at base; leaves $10-20 \times 4.5-$

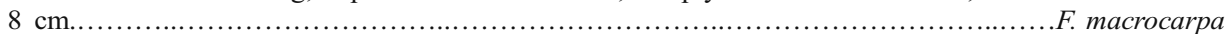

11. Pedicels $0.8-1.5 \mathrm{~cm}$; tepals $1-1.8 \times 0.4-0.7 \mathrm{~cm}$, slightly constricted at base; leaves $5-13 \times 2-$

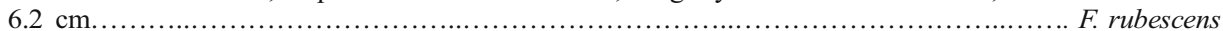

10. Midrib plane or prominulous on upper leaf surface.

12. Pedicels 3-4 cm long; plants of Tanzania, Mauritius, or Rodrigues.

13. Tepals $1.7-2 \times 0.8-0.9 \mathrm{~cm}$; disc subquadrangular, $4 \mathrm{~mm}$ diam.; plants of Tanzania............ africana

13. Tepals $2.2-2.5 \times 0.8-1.8 \mathrm{~cm}$; disc circular, $8 \mathrm{~mm}$ diam.; plants of Rodrigues or Mauritius.

14. Leaves strongly asymmetric; margins revolute; tepals chartaceous, triangular-acute at apex, fused at base, not reflexed; plant of Rodrigues.................................... rodriguesiana

14. Leaves not asymmetric; margins not revolute; tepals fleshy, oblong-lanceolate, acuminate at apex, obtuse or rounded at summit, free to base, reflexed; plant of Mauritius. .F. mauritiana

12. Pedicels $0.3-2.5 \mathrm{~cm}$ long; plants of Madagascar.

15. Leaves $9-19 \times 3.7-7 \mathrm{~cm}$; pedicels $1.5-2.3 \mathrm{~cm}$ long.

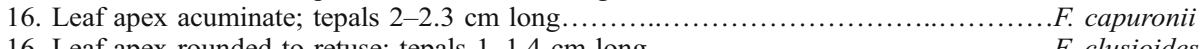

16. Leaf apex rounded to retuse; tepals $1-1.4 \mathrm{~cm}$ long................................. clusioides

15. Leaves $1.2-6.5 \times 0.4-2.5 \mathrm{~cm}$; pedicels $0.3-1 \mathrm{~cm}$ long.

17. Leaves slightly asymmetric, narrowly lanceolate-obovate; tepals elliptic, $1.5-1.8 \times 0.4-0.6 \mathrm{~cm}$ F. dracaenoides

17. Leaves markedly asymmetric, oblong to oblanceolate; tepals lanceolate, $1.7-2.0 \times 0.3-0.4 \mathrm{~cm}$

.F. asymmetrica

Foetidia africana Verdc., Kew Bull. 40: 635. 1985. Type: Tanzania. Uzaramo district: $80 \mathrm{~km}$ W of Dar es Salaam, Dar-Morogoro road, Vigwasa, 80 m, 5 Dec 1978 (fl), L. B. Mwasumbi 11607 (holotype: K; isotypes: DSM, K).

Small tree to $8 \mathrm{~m}$ tall. Leaves subsessile, oblong-elliptic to obovate, 4.5-9.2 $\times 2.3-$ $4.5 \mathrm{~cm}$; apex rounded or slightly retuse in some leaves, base cuneate, equal; midrib plane to prominulous above, slighty excentric. Flowers not seen. Fruit solitary, axillary; pedicels to $3.5 \mathrm{~cm}$ long, with two small bracteoles at junction with receptacle, the bracteoles 1.5-3 mm long, lanceolate; receptacle turbinate, $3.75 \mathrm{~mm}$ in young fruit; tepals $1.7-2 \times 0.8-0.9 \mathrm{~cm}$, narrowly oblong-lanceolate, chartaceous, bluntly acute at apex; style 1-1.3 cm long; disc subquadrangular, $4 \mathrm{~mm}$ broad.
Distribution and habitat.-Tanzania, riverine forest in lowlands.

This species is known only from the type collection.

Foetidia asymmetrica $H$. Perrier, as "asymetrica", Notul. Syst. (Paris) 14: 305. 1953. Type: Madagascar. NE of Maevatanana (Boina) near Tainangidina, 1905 (fr), H. Perrier de la Bâthie 6527 (holotype: P).

Small to large tree to $20 \mathrm{~m}$ tall. Leaves subsessile, oblong to oblanceolate, 2.5-6.5 $\times$ 1-2.5 cm; apex rounded or retuse, base cuneate, markedly asymmetric; midrib plane above, markedly excentric. Flowers not seen. Fruit solitary, axillary; pedicels ca. $0.8 \mathrm{~cm}$ long, ebracteolate when fruiting; receptacle turbinate, $1-1.5 \mathrm{~cm}$; tepals $1.7-2 \times 0.3-$ $0.4 \mathrm{~cm}$, lanceolate, chartaceous, acute at 
apex; style persistent, $1.3 \mathrm{~mm}$ long; disc circular, $5 \mathrm{~mm}$ diam.

Distribution and habitat.-Sandy and calcareous soil, lowlands up to $1000 \mathrm{~m}$, widespread in northern and western Madagascar (Fig. 2C).

\section{Local name.-Taimpapango.}

Selected specimens examined. MADAGASCAR. Antisiranana: Sahafary, $12^{\circ} 34^{\prime} \mathrm{S}, 49^{\circ} 26^{\prime} \mathrm{E}, 25 \mathrm{Apr}$ 1963, Capuron SF22714 (K, MO, P); Mahajanga, Bemaraha Reserve, $18^{\circ} 12^{\prime} \mathrm{S}, 44^{\circ} 35^{\prime}-4^{\circ} 58^{\prime} \mathrm{E}, 100$ 150 m, 6-12 Dec 1952, Leandri et al. 2142 (K, MO). Toamasina: Perinet-Analamazoatra Reserve, $18^{\circ} 56^{\prime} \mathrm{S}$, $47^{\circ} 26^{\prime} \mathrm{E}, 1000 \mathrm{~m}, 1919$, Thouvenot 139 (TAN). Toliara: Kirindy, $20^{\circ} 04^{\prime} \mathrm{S}, 44^{\circ} 40^{\prime} \mathrm{E}, 35 \mathrm{~m}, 14-16$ Mar 1992 , Noyes et al. 1019 (MO).

All the specimens studied are in fruit or young fruit.

Foetidia capuronii Bosser, Adansonia, 10: 112. 1988. Type: Madagascar. Antisiranana: forêt d'Andrakaraka near Antalaha, 18 Apr 1966 (young fr), R. Capuron SF24634 (holotype: P; isotypes: P, TEF).

Small tree to $8 \mathrm{~m}$ tall. Leaves shortly petiolate to subsessile, the petiole $0-3 \mathrm{~mm}$ long; lamina papyraceous, elliptic to obovate, $9-19 \times 3.7-7 \mathrm{~cm}$, apex shortly acuminate, the acumen 5-10 mm long, base cuneate, slightly asymmetric, midrib plane or prominulous above. Flowers not seen. Fruit solitary, axillary; pedicels $1.5-2.5 \mathrm{~cm}$ long, with 2 bracteoles at junction with receptacle or slightly below; bracteoles linear, ca. $3 \mathrm{~mm}$ long; receptacle obpyramidal, $5 \mathrm{~mm}$ tall, slightly 4-winged; tepals lanceolate, 2-2.3× $0.7-1 \mathrm{~cm}$, acute at apex; style persistent, ca. $1 \mathrm{~cm}$ long, 4-fid; disc flat, $4 \mathrm{~mm}$ diam., circular.

Distribution and habitat.-Antisiranana Province, Madagascar, lowland forest (Fig. 2A).

Additional specimen examined. MADAGASCAR. Antisiranana: Ambatobiribiry, $14^{\circ} 10^{\prime} 53^{\prime \prime} \mathrm{S}, 50^{\circ} 5^{\prime} 5^{\prime \prime} \mathrm{E}$, 100 m, 25 Mar 2001, Rabenantoandro et al. 465 (TAN).

This species is distinguished by the large, thin chartaceous leaves. Bosser (1988) notes that it grows in the same habitat as $F$. parviflora (flowering in September) but flowers at a different time of year (April).
Foetidia clusioides Baker, Kew Bull. 1985: 104.1985.Type: Madagascar. North, without locality (fl), R. Baron 6250 (holotype: $\mathrm{K}$; isotype: $\mathrm{BM})$.

Tree to $15 \mathrm{~m}$ tall. Leaves sessile, coriaceous, oblong-lanceolate, $9-13 \times 4-6.2 \mathrm{~cm}$, apex rounded, base cuneate; midrib plane above, slightly curved to one side, with strong marginal vein. Flowers solitary, axillary; pedicels ca. $2.2 \mathrm{~cm}$ long, ebracteolate; tepals oblong-oblanceolate, stamens many, arranged in four groups, shorter than style; $1-1.4 \mathrm{~cm}$ long, acute at apex; style ca. $0.8 \mathrm{~cm}$ long, 4-fid at apex; disc flat, ca. $4 \mathrm{~cm}$ diam., circular. Fruit not seen.

Distribution and habitat. - Northern Madagascar, lowlands to $1000 \mathrm{~m}$ (Fig. 2B).

Selected specimens examined. MADAGASCAR. Antisiranana: Montagne des Français, $12^{\circ} 22^{\prime} 18^{\prime \prime} \mathrm{S}, 49^{\circ}$ $20^{\prime} 39^{\prime} \mathrm{E}, 300$ m, 1 Apr 2004, Ratovoson et al. 746 (CNARP, MO, P, TAN). Toamasina: AmbilaLemaitso, $18^{\circ} 49^{\prime} \mathrm{S}, 49^{\circ} 8^{\prime} \mathrm{E}, 30 \mathrm{~m}, 10$ Aug 1957, Capuron SF18065 (MO, P).

Foetidia cuneata Bosser, Adansonia 10: 110. 1988. Type: Madagascar. Toamasina: Ana-

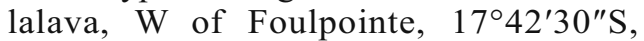
49 $27^{\prime} \mathrm{E}, 20$ Nov 1964, (fr), R. Capuron SF23852 (holotype: P; isotypes: G, K, MO, $\mathrm{P}, \mathrm{TEF})$.

Small to medium tree 4-20 m. Leaves shortly petiolate; petioles $1-3 \mathrm{~mm}$ long; lamina thickly coriaceous, drying grey above, reddish-brown beneath, cuneiform, slightly asymmetric, broader near apex, $1.3-4 \times 0.5-$ $2 \mathrm{~cm}$, apex retuse, base cuneate; midrib slightly impressed above. Flowers not seen. Fruit solitary, axillary; pedicels $1-1.5 \mathrm{~cm}$ long, with two bracteoles at junction with receptacle; bracteoles linear, 2-4 $\mathrm{mm}$ long; receptacle obconical, $1-1.5 \mathrm{~cm}$ long; tepals triangular, $0.4-0.7 \times 0.2-0.25 \mathrm{~cm}$, subacute at apex, reflexed in fruit; style base persistent; disc circular, conical, $1.5-2 \mathrm{~mm}$ diam.

Distribution and habitat.-Northeastern Madagascar, lowland forest on laterite soils, 0-50 m (Fig. 3A).

Additional specimens examined. MADAGASCAR. Antisiranana: Anjia, $15^{\circ} 16^{\prime} 18^{\prime \prime} \mathrm{S}, 50^{\circ} 20^{\prime} 35^{\prime \prime} \mathrm{E}, 36 \mathrm{~m}, 23$ Feb 2001, Razakamalala et al. 64 (MO, P, TAN). 
Toamasina: Masoala PN, $15^{\circ} 47^{\prime} \mathrm{S}, 50^{\circ} 2^{\prime} \mathrm{E}, 0 \mathrm{~m}, 20 \mathrm{Nov}$ 1994, Rahajasoa et al. 947 (TAN).

This species is similar to F. parviflora and $F$. delphinensis in the small leaves, but differs in the solitary flowers with larger tepals and the larger fruit.

Foetidia delphinensis Bosser, Adansonia 10: 108. 1988. Type: Madagascar. Tôlanaro: Fort Dauphin, Forêt d'Andrazato, $5 \mathrm{Apr}$ 1954 (fr), Service Forestier du Madagascar, without collector SF9739 (holotype: P; isotypes: $\mathrm{P}, \mathrm{TEF})$.

Tree to $20 \mathrm{~m}$ tall. Leaves subsessile or shortly petiolate, finely papillate, drying greynitid, the petiole 1-5 $\mathrm{mm}$ long; lamina coriaceous, broadly elliptic to oval, symmetrical, $2.5-5.5 \times 1.3-3.2 \mathrm{~cm}$, apex obtuse or rounded, base cuneate; midrib impressed above. Flowers borne in axillary fascicles of $2-4$, not seen. Fruit $2-2.5 \mathrm{~cm}$ long; pedicels thick and rigid, ca. $2 \mathrm{~cm}$ long, with 2 bracteoles at junction with receptacle; bracteoles linear, 4-5 mm long; receptacle obconical, ca. $1 \mathrm{~cm}$ long, without ridges; tepals oval, $1 \times 0.4-0.5 \mathrm{~cm}$, obtuse or rounded at apex, strongly reflexed in fruit; persistent style $0.8-0.9 \mathrm{~cm}$ long; disc circular, $4 \mathrm{~mm}$ diam.

Distribution and habitat.-The only species found in the extreme southeast of Madagascar, lowland forest, $0-500 \mathrm{~m}$ (Fig. 2A).

$$
\text { Local name.-Rangomafitry. }
$$

Additional specimen examined. MADAGASCAR. Tôlanaro: Bermangidy, $100-600 \mathrm{~m}, 24^{\circ} 34^{\prime} \mathrm{S}, 47^{\circ} 12^{\prime} \mathrm{E}$, 31 Mar 1955, Bégué SF13194 (P).

Only three other species of Foetidia ( $F$. retusa, $F$. obliqua and $F$. parviflora) have flowers borne in clusters rather than solitary. Foetidia delphinensis differs from F. parviflora in the bracteoles inserted at the base of the receptacle, the larger fruit with reflexed tepals and the papillate leaves. It differs from $F$. retusa in the tendency to larger leaves, with longer petioles, and the midrib plane (vs. impressed), and from $F$. obliqua in the petiolate, oblong leaves with more acute apices.
Foetidia dracaenoides Capuron ex Bosser, Adansonia 10: 106. 1988. Type: Madagascar. Antisiranana: Montagne des Français, Vallon de 1'Andavakoera, Diégo Suarez, $1000 \mathrm{~m}, 12^{\circ} 22^{\prime} \mathrm{S}, 4^{\circ} 21^{\prime} \mathrm{E}, 20$ Dec 1963 (fl), R. Capuron SF23005 (holotype: P; isotypes: $\mathrm{B}, \mathrm{BR}, \mathrm{G}, \mathrm{L}, \mathrm{MO}, \mathrm{P}, \mathrm{PRE}$, TEF, WAG).

(Figs. 1A-F)

Shrub or small tree to $8 \mathrm{~m}$ tall. Leaves sessile, coriaceous, narrowly lanceolate-obovate, slightly asymmetric, $1.2-6 \times 0.4-$ $1.7 \mathrm{~cm}$, apex rounded or minutely apiculate, base rounded; midrib prominulous above. Flowers axillary, solitary; pedicels puberulous, $0.3-0.8 \mathrm{~cm}$ long, with two bracteoles at junction with receptacle; bracteoles linear, 4$7 \mathrm{~mm}$ long; tepals elliptic, acute at apex, 1.5$1.8 \times 0.4-0.6 \mathrm{~cm}$; stamens ca. 70, 5-12 mm long; ovary 2-locular, subquadrate, with 8-12 ovules in each loculus; style $1-1.3 \mathrm{~cm}$ long, bifid at apex; disc circular, 4-5 $\mathrm{mm}$ diam. Mature fruit not seen, young fruit tetragonal, 5.5-6 mm diam.

Distribution and habitat.-Extreme north of in Antisiranana Province, Madagascar, forest on calcareous soils up to $1000 \mathrm{~m}$. Fig. 3B

Selected specimens examined. MADAGASCAR. Antisiranana: Analamera RS, $127 \mathrm{~m}, 12^{\circ} 40^{\prime} \mathrm{S}, 4^{\circ} 32^{\prime}$ 43"E, 17 Jan 1995, Andrianantoanina et al. 741 (MO, NY, P, TAN); Montagne des Français, $1000 \mathrm{~m}, 12^{\circ} 22^{\prime} \mathrm{S}$ 49 $22^{\prime}$ E, 28 Jan 1966, Capuron, SF24429 (K, MO).

The leaves are clustered at the apex of the branches giving a pachycaul appearance that distinguishes this species from most others.

Foetidia macrocarpa Bosser, Adansonia 10: 117. 1988. Type: Madagascar. Mahajanga: Befotaka, NE of Autsohihy road to Maromandia, $14^{\circ} 32^{\prime} \mathrm{S}, 48^{\circ} 1^{\prime} \mathrm{E}, 6$ Nov 1956 (fr), $R$. Capuron SF18883bis (holotype: P; isotypes: BR, G, K, MO, P, TEF, WAG).

Small tree 4-12 m tall. Leaves sessile, tapering to narrow almost winged petiole-like structure at base, chartaceous, elliptic to obovate, symmetrical, $10-20 \times 4.5-8 \mathrm{~cm}$, apex rounded to acuminate, the acumen 0 $8 \mathrm{~mm}$ long, base cuneate; midrib slightly impressed above. Flowers not seen. Fruit 

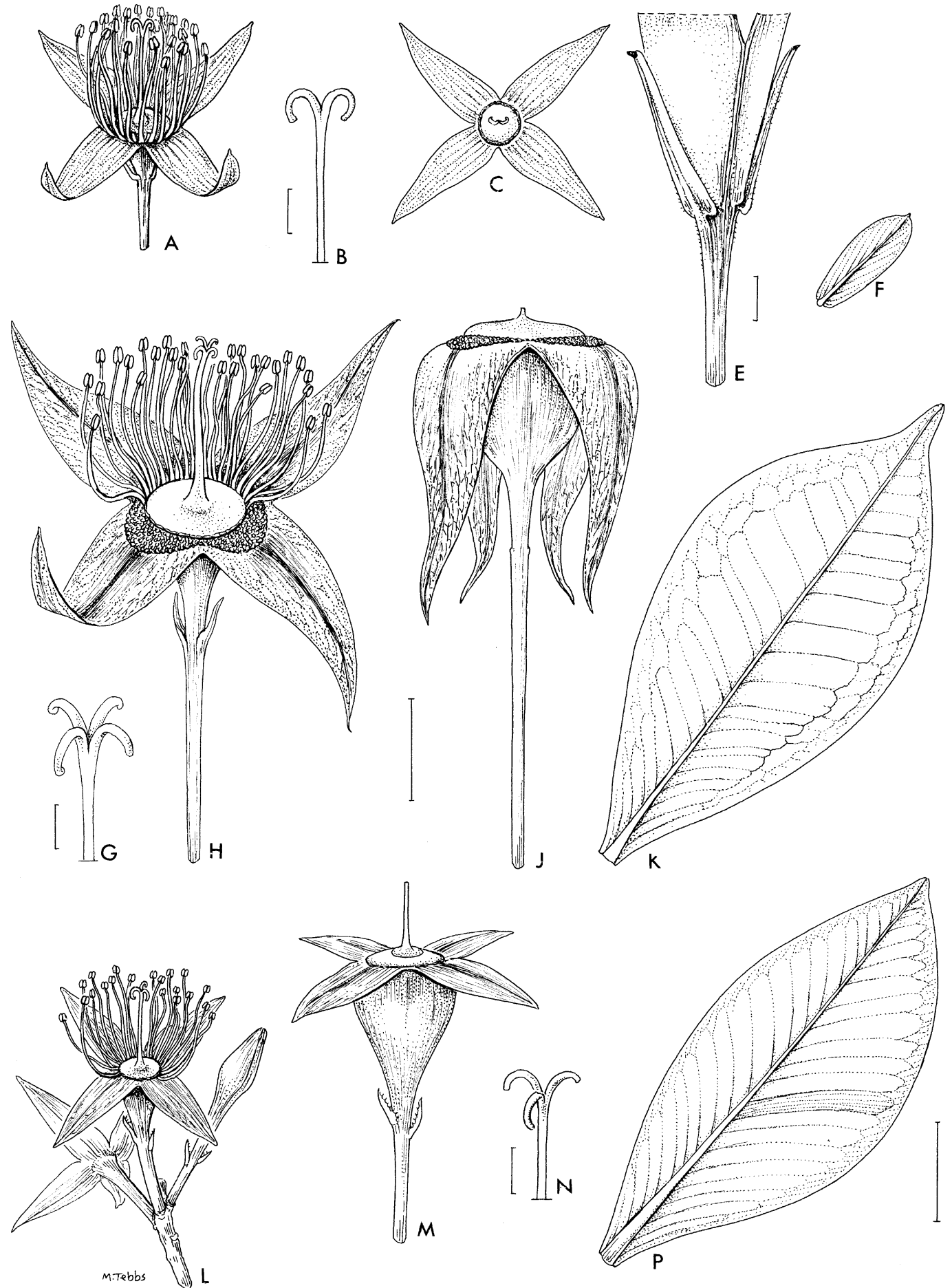

Fig. 1. Species of Foetidia. A-F. F. dracaenoides. A. Flower. B. Style. C. Persistent tepals. E. Bracteoles. F. Leaf. G-K. F. mauritiana. G. Style. H. Flower. J. Fruit and persistent tepals. K. Leaf. L-P. F. obliqua: L. Flowers. M. Young fruit and tepals. N. Style. P. Leaf. (A-F, Capuron SF23005; G \& H, Cadet 4977; J \& K, Cadet 3710; L \& N, Gereau et al. 3285; M \& P, Schatz et al. 1795). Drawn by Margaret Tebbs. 


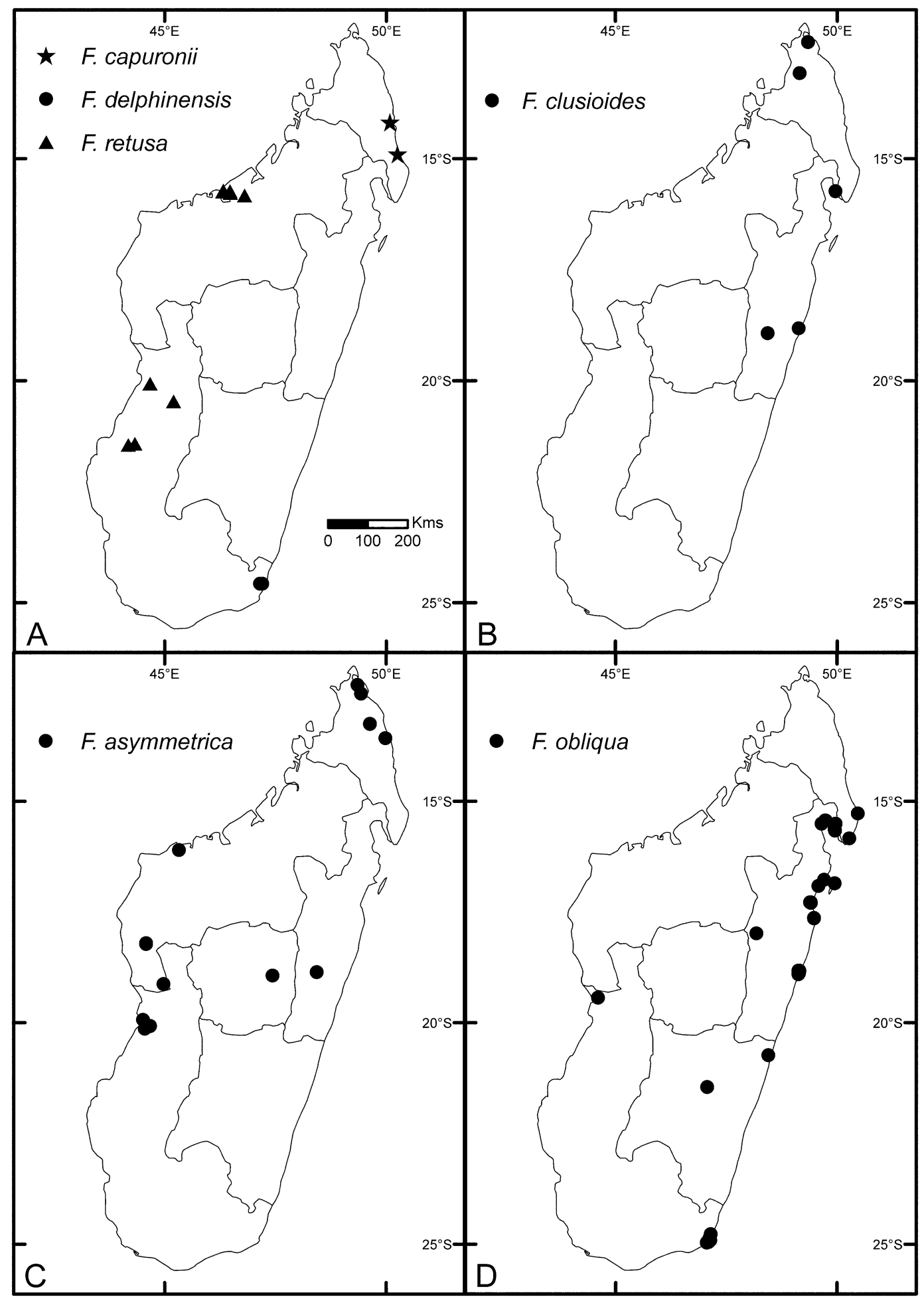

Fig. 2. Distributions of species of Foetidia. A. F. retusa, triangle; F. delphinensis, circle; F. capuronii, star. B. F. clusioides. C. F. asymmetrica. D. F. obliqua. 


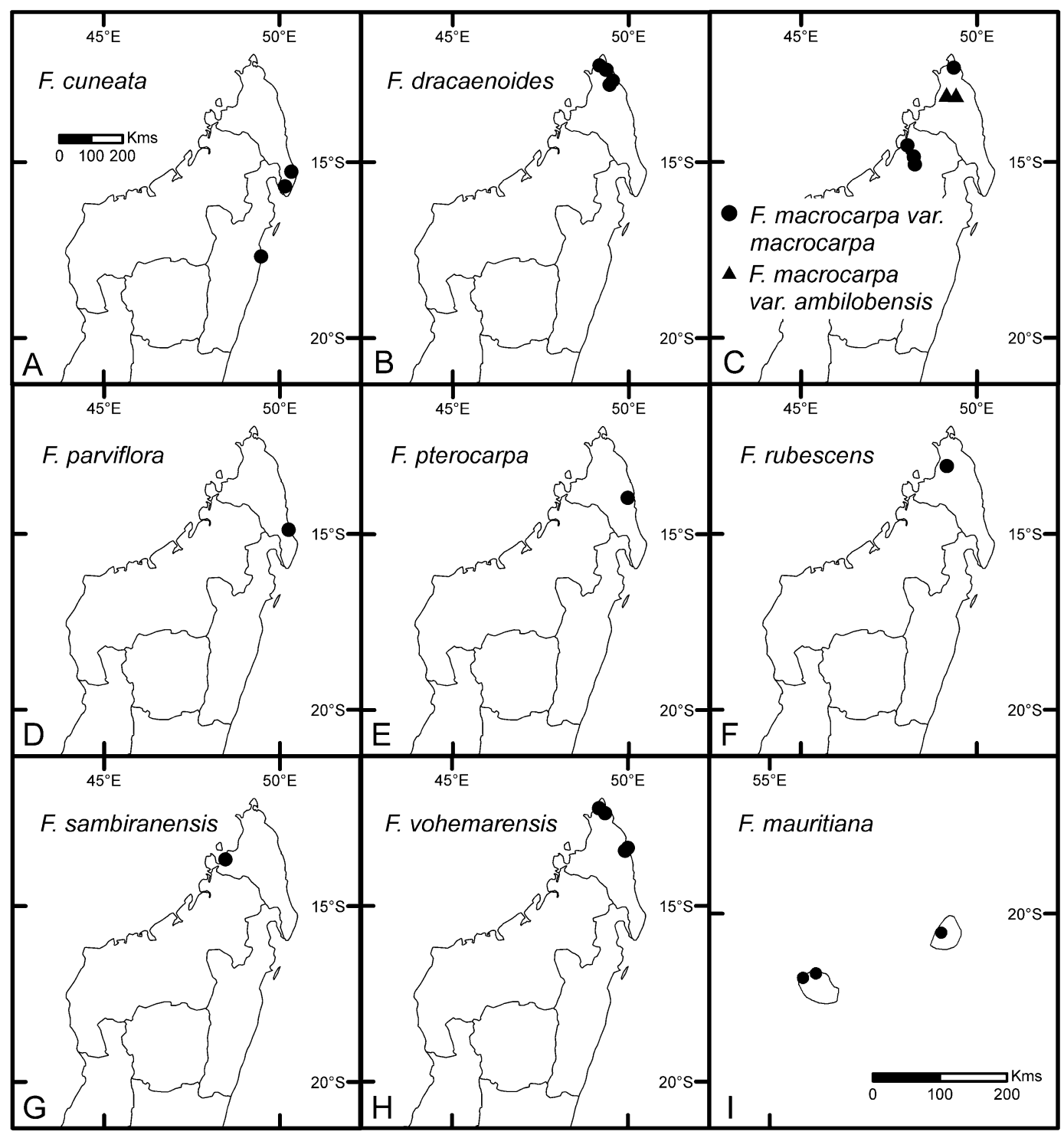

Fig. 3. Distributions of species of Foetidia. A. F. cuneata. B. F. dracaenoides. C. F. macrocarpa var. macrocarpa, circles; F. macrocarpa var. ambilobensis, triangle. D. F. parviflora. E. F. pterocarpa. F. F. rubescens. G. F. sambiranensis. H. F. vohemarensis. I. F. mauritiana from Mauritius and Réunion.

axillary, solitary; pedicels $4-6.5 \mathrm{~cm}$ long; bracteoles 2, inserted on base of receptacle, $0.5-1 \mathrm{~cm}$ long, linear, persistent; receptacle obconical, $1.5 \mathrm{~cm}$ long; tepals coriaceous, broadly oval, $3.5-5.5 \times 2.5-4 \mathrm{~cm}$ to lanceolate $4-5 \times 2-2.7 \mathrm{~cm}$, constricted at base, rounded to acute at apex; style persistent, $1.2-1.3 \mathrm{~cm}$ long, 4-fid; disc quadrangular, $1 \mathrm{~cm}$ broad.

\section{Key to varieties of $\boldsymbol{F}$. macrocarpa}


Foetidia macrocarpa Bosser var. macrocarpa

Distribution and habitat.-Northeastern Madagascar, dry lowland forest to $500 \mathrm{~m}$ (Fig. 3C).

Additional specimens examined. MADAGASCAR. Mahajanga: Bora, $100-411 \mathrm{~m}, 14^{\circ} 51^{\prime} \mathrm{S}, 48^{\circ} 12^{\prime} \mathrm{E}, 23 \mathrm{Jul}$ 1958, Capuron SF 18075 (K, MO, TEF); Ampombilava, $15^{\circ} 4^{\prime} \mathrm{S}, 48^{\circ} 14^{\prime} \mathrm{E}, 16$ Mar 1965, Capuron SF 24069 (TEF).

Foetidia macrocarpa Bosser var. ambilobensis Bosser, Adansonia 10: 119. 1988. Type: Madagascar. Antisiranana: Ankaramy, canton de Betsiaka, Ambilobe Distr., Road to Vohémar, $13^{\circ} 6^{\prime} \mathrm{S}, 4^{\circ} 24^{\prime} \mathrm{E}$ (fr), 23 May 1958, Service Forestier du Madagascar without collector SF19548 (holotype: $\mathrm{P}$; isotypes: $\mathrm{G}, \mathrm{K}, \mathrm{MO}, \mathrm{P}, \mathrm{TEF}$ ).

Small tree to $5 \mathrm{~m}$ tall. Differs from Foetidia macrocarpa var. macrocarpa in the lanceolate tepals, $4-5 \times 2-2.7 \mathrm{~cm}$, and the apex acute to subacute.

Distribution and habitat.-Northern Madagascar, dry lowland forest (Fig. 3C).

Additional specimens examined. MADAGASCAR. Antisiranana: Marivorahona, $100 \mathrm{~m}, 13^{\circ} 5^{\prime} 27^{\prime \prime} \mathrm{S}, 4^{\circ} 7^{\prime}$ 58"E, 18 Jan 2005, Rakotondrajaona et al. 354 (CNARP, MO, P, TAN).

Foetidia mauritiana Lam., Encycl. 2 (2): 457. 1788. Type: Mauritius. Ile de France (fl), P. Commerson s.n. (holotype: P-LA; isotypes: P-JU 13976, P). (Figs. 1G, K)

Foetidia borbonica J. F. Gmel., Syst. Nat., ed. 13[bis], 2 (1): 793. 1791. Type: probably 1769, Commerson s.n. (P) from Mauritius, locality unknown.

Foetidia mauritiana Lam. var. elongata Knuth, Pflanzenr. (Engler) IV. 219: 63. 1939. Type: India; Cultivated in Calcutta Botanic Garden: W. Griffith 2420/21 (possible isotype: $\mathrm{K}$, see note).

Tree or shrub to $7 \mathrm{~m}$ tall. Leaves sessile, coriaceous, elliptic, $8-13 \times 3.5-6.3 \mathrm{~cm}$, symmetric, the margins entire, apex acute to shortly acuminate, base cuneate; midrib plane above. Flowers solitary, axillary; pedicels ca. $3 \mathrm{~cm}$ long; bracteoles inserted at base of petiole, $3 \mathrm{~mm}$ long; receptacle turbinate; tepals oblong-lanceolate, $2.2-2.5 \mathrm{~cm}$ long, fleshy, free to base, acuminate at apex; stamens numerous, grouped in clusters, 2.2$2.8 \mathrm{~cm}$ long, style equaling stamens in length, 4-fid. Fruit pedicels to $4 \mathrm{~cm}$ long; disc circular to slightly quadrate, $10 \mathrm{~mm}$ diam.

Distribution and habitat.-Confined to Mauritius and Réunion in the Mascarenes, in semi-deciduous dry forest and hill forest to 500 m. Fig. 3I.

Local name.-Bois puant.

Selected specimens examined. MAURITIUS. Trois Mamelles, 14 Feb 1968, Capuron SF28269 (K, P); Yemen Valley, 22 m, 4 Jul 1979, Lorence 2698 (K). RÉUNION. Vallée de la Riv. des Galets, 30 Jan 1975, Cadet 3710 (K, P).

Even in 1909 the collection F. Bijou s.n. (BISH) noted that this is "a most valuable timber tree now becoming rare". It was formerly cultivated at the Calcutta Botanic Garden. The specimen Griffith s.n. 1845, K, from Calcutta is possibly an isotype of $F$. mauritiana var. elongata of Knuth (1939).

Foetidia obliqua Blume, Mus. Bot. Lugd.Bat. 1:145. 1850. Type: Madagascar. Without collector s.n. (holotype: P, n.v.). (Figs. 1L-P)

Shrub or small tree to $9 \mathrm{~m}$ tall. Leaves sessile, coriaceous, asymmetrical, oblong, $6.5-9.5 \times 2.5-3.8 \mathrm{~cm}$, apex rounded to bluntly acute, base cuneate; midrib asymmetric, plane above. Flowers axillary and terminal, in clusters of $3-5$; pedicels ca. $1 \mathrm{~cm}$ long, with two bracteoles just below junction with receptacle; bracteoles lanceolate, 2-3 mm long; receptacle turbinate, $1.3-1.5 \mathrm{~cm}$ long; tepals lanceolate, $1-1.2 \mathrm{~cm}$ long, acute at apex; stamens numerous; style equaling stamens, $1 \mathrm{~cm}$ long, 3-lobed; disc circular, slightly raised, $5 \mathrm{~mm}$ diam. Fruit turbinate.

Distribution and habitat.-The commonest and most widespread species of Foetidia in Madagascar, Forest on sandy soil, littoral to $1400 \mathrm{~m}$ (Fig. 2D).

Local name.-Namoloana.

Selected specimens examined. MADAGASCAR. Antisiranana: Masoala PN, $20 \mathrm{~m}, 15^{\circ} 16^{\prime} 23^{\prime \prime} \mathrm{S}, 50^{\circ} 28^{\prime}$ 
18" E, 15 May 1999, Randriatafika et al. 45 (MO, P, TAN); Fianarantsoa, Ampasimaneva, 13 m, 2043'59" S 48 27'9" E, 15 Jun 2004, Razakamalala et al. 1488 (MO, P, TEF). Toamasina: Ambila-Lemaitso, $5 \mathrm{~m}, 18^{\circ} 49^{\prime} \mathrm{S}$, 49 $9^{\prime} \mathrm{E}, 16$ May 1991, Zarucchi et al. 7430 (K, MO). Toliara: Sainte Luce, $20 \mathrm{~m}, 24^{\circ} 52^{\prime} \mathrm{S}, 4^{\circ} 7^{\prime} \mathrm{E}, 13$ Mar 1989, Gereau et al. 3315 (K, MO).

The collection Vaughan 651 (EA, FHO) from Choke Creek, Pemba, off the coast of Tanzania, is definitely this species. It was probably introduced to Pemba and it matches well with the Madagascan material. I have not yet been able to determine which specimen at Paris is the type used by Blume. There are 13 sheets old enough to have been studied by Blume. Blume described stamens and anthers and none of these sheets studied have those parts so either it is another specimen not seen or the stamens have fallen off as is frequent in Foetidia.

Foetidia parviflora Capuron ex Bosser, Adansonia 10: 108. 1988. Type: Madagascar. Antisiranana: Forêt d' Andrazato, SW of Antalaha, 21 Sep 1954 (fl), R. Capuron SF11236 (holotype: P; isotypes: K, MO, P, TEF).

Large tree with scaly bark. Leaves petiolate, the petioles 3-12 $\mathrm{mm}$ long; lamina coriaceous, ovate to obovate, symmetrical, $2.5-6 \times 1.3-4 \mathrm{~cm}$, apex usually retuse, or less often rounded, base cuneate; midrib impressed above. Flowers borne in axillary and terminal fascicles of $2-5,1$ cucullate bract $2-2.5 \mathrm{~mm}$ long, caducous; pedicels $2-2.5 \mathrm{~cm}$ long at anthesis, with two bracteoles at base of pedicel; bracteoles lanceolate, 2-3 mm long; receptacle conical, ca. $5 \mathrm{~mm}$ long, slightly 4 winged; tepals (3) 4 , thick, ovate, $0.3-0.4 \times$ 0.2. $-0.25 \mathrm{~cm}$ long, rounded to acute at apex; reflexed; stamens ca. 50, 1.5- $3 \mathrm{~mm}$ long; ovary 2-locular, with 5-7 ovules in each loculus; style $0.3-0.4 \mathrm{~cm}$ long, 2 or 3-fid; disc hemispherical, 3-4 $\mathrm{mm}$ diam. Fruit $0.8-$ $1.6 \mathrm{~cm}$ long,, reflexed;

Distribution and habitat.-Known only from the type specimen from Antisiranana, lowland rainforest at $90 \mathrm{~m}$ (Fig. 3D).

This is a most distinct species on account of the very small flowers borne in clusters of
$2-5$, the small fruit, and the bracteoles inserted at the base of the pedicels.

Foetidia pterocarpa Bosser, Adansonia 10: 111. 1988. Type: Madagascar. Antisiranana: South of Antsirabe Nord, road Vohémar-Sambava, $13^{\circ} 58^{\prime} \mathrm{S}, 49^{\circ} 58^{\prime} \mathrm{E}, 21$ Oct 1966 (fl), R. Capuron SF24896 (holotype: P; isotypes: $\mathrm{G}, \mathrm{K}, \mathrm{MO}, \mathrm{P}, \mathrm{TEF}$ ).

Large tree to $25 \mathrm{~m}$ tall. Leaves shortly petiolate, the petiole 3-6 $\mathrm{mm}$ long; lamina coriaceous, obovate, slightly asymmetric, $2.5-6 \times 1.3-3 \mathrm{~cm}$, apex obtuse to slightly acute, base cuneate; midrib impressed above. Flowers not seen. Fruit axillary, solitary; pedicels ca. 0.4-1.1 cm long, with two bracteoles inserted just below junction with receptacle; bracteoles linear, 5-10 mm long; receptacle obconical, $0.8-1 \mathrm{~cm}$ tall, distinctly quadrangular and slightly winged; tepals ovate, $0.8-1.2 \times 0.5-0.8 \mathrm{~cm}$, acute to rounded at apex; style persistent, $0.7-0.9 \mathrm{~cm}$ long, 4fid; disc circular, $4 \mathrm{~mm}$ diam.

Distribution and habitat.-Known only from the type collection from northeastern Madagascar, lowland rainforest, 0-100 m (Fig. 3E).

This species is distinguished by the shallow wings on the receptacle.

Foetidia retusa Blume, Mus. Bot. Lugd.-Bat. 1: 144. 1849. Type: Madagascar. Ambongo, 1841 (young fr), A. Pervillé $669 a$ (holotype: $\mathrm{P}$; isotypes: $\mathrm{K}, \mathrm{MO}$ ).

Shrub or small tree to $10 \mathrm{~m}$ tall. Leaves petiolate, the petioles 5-14 mm long; lamina coriaceous, broadly obovate, symmetrical, 2.5-9.5 × 1.4-6 cm, apex deeply retuse, base broadly cuneate; midrib plane above. Flowers in terminal clusters of $2-6$; pedicels $0.8-1 \mathrm{~cm}$ long with two bracteoles at junction with receptacle; bracteoles linear, 6-10 $\mathrm{mm}$ long; receptacle broadly campanulate, ca. $8 \mathrm{~mm}$ long; tepals $1.4-1.6 \mathrm{~cm}$ long, lanceolate, acute at apex; stamens numerous, ca. $15 \mathrm{~mm}$ long; style $0.4-0.5 \mathrm{~cm}$, shortly 4-fid; disc circular, $6 \mathrm{~mm}$ diam. Fruit campanulate, pedicels $0.8-1 \mathrm{~cm}$ long. 
Distribution and habitat.-Eastern Madagascar, in savanna on limestone, savanna margins and seasonally flooded savanna 0 $400 \mathrm{~m}$ (Fig. 2A).

Local name.-Namolona (SF14108).

Selected specimens examined. MADAGASCAR. Mahajanga: Majunga, 0-50 m, $15^{\circ} 43^{\prime} \mathrm{S}, 46^{\circ} 19^{\prime} \mathrm{E}$, Jun 1953, Bosser 5832 (MO, TAN). Toliara: Ambohibe, 240 m, $20^{\circ} 28^{\prime} \mathrm{S}, 45^{\circ} 12^{\prime} \mathrm{E}, 18 \mathrm{Mar} 1971$, Mabberley 736 (K, TAN).

Foetidia rodriguesiana F. Friedmann, Adansonia 20: 448. 1981 Type: Mascarenes: Rodrigues, Cascade St. Louis, 15 Mar 1978 (fl), F. Friedmann 3377 (holotype: P; isotype: $\mathrm{K})$.

Tree to $10 \mathrm{~m}$ with tortuous trunk. Leaves sessile, coriaceous, elliptic to obovate, asymmetric, $4.5-11 \times 2-3.3 \mathrm{~cm}$, apex rounded, base cuneate, the margins slightly revolute; midrib plane above. Flowers axillary, solitary; pedicels 3-4 cm long, with two bracteoles inserted at junction with receptacle; bracteoles linear, 4-6 mm long; receptacle campanulate, not ribbed; tepals $2.3 \times 0.8-1.8 \mathrm{~cm}$, chartaceous, rounded at apex, slightly fused at base, not reflexed; stamens numerous, arranged in four groups, ca. $15 \mathrm{~cm}$ long; ovary 4-locular with 20 ovules in each loculus; style 1-1.5 cm long, 2- or 4-fid; disc circular, swollen, $8 \mathrm{~mm}$ diam. Fruit $6-8 \mathrm{~cm}$, with 4 persistent aliform tepals.

Distribution and habitat.-Confined to forest remnants on Rodrigues island.

Selected specimens examined. RODRIGUES ISLAND. Vallée Grand Baie, Jul 1976, Cadet 2435 (P, REU); without locality, 1874, Balfour s.n.(K), 1864-65, Bouton s.n.(K).

This species is close to $F$. mauritiana, but differs in the thinner chartaceous tepals that are united at the base and not reflexed in fruit, the less acute leaves with slightly revolute margins, and the absence of a glandular area at the base of the tepals. The protologue stated that there were not more than twenty trees of this species remaining at the time of collection of the type specimen.

Foetidia rubescens Bosser, Adansonia 10: 112. 1988. Type: Madagascar: Antisiranana base of Ambohibe, east of Merivorahona, $13^{\circ} 4^{\prime} \mathrm{S}, 4^{\circ} 9^{\prime} \mathrm{E}, 3$ May 1966 (fr), $R$. Capuron SF24740 (holotype: P; isotypes: MO, P, TEF).

Small tree. Leaves sessile, chartaceous, asymmetric, obovate, 5-13×2-6.2 cm, apex rounded or shortly acuminate, base cuneate; midrib slightly impressed above, asymmetric. Flowers not seen. Fruit axillary, solitary; pedicels $0.8-1.5 \mathrm{~cm}$ long; bracteoles 2 , inserted on base of receptacle, linear to narrowly triangular, $2 \mathrm{~mm}$ long; receptacle obconical, ca. $4 \mathrm{~mm}$ tall, not ribbed; tepals lanceolate, coriaceous, $1-1.8 \times 0.4-0.7 \mathrm{~cm}$, acute at apex, slightly constricted at base; style base persistent in fruit; disc quadrangular, $4 \mathrm{~mm}$ broad.

Distribution and habitat.-Known only from the type specimen from northern Madagascar, dry forest (Fig. 3F).

This species is perhaps close to $F$. capuronii, but differs in the sessile, smaller leaves, and the short stubby tepals with rounded apices.

Foetidia sambiranensis Bosser, Adansonia 10: 116. 1988. Type: Madagascar. Antisiranana: Vallée de la Beandrone, $\mathrm{E}$ of Ambanja, $13^{\circ} 40^{\prime} \mathrm{S}, 48^{\circ} 27^{\prime} \mathrm{E}, 29$ Oct 1954 (fr), R. Capuron SF11390 (holotype: P; isotypes: $\mathrm{G}, \mathrm{K}, \mathrm{MO}, \mathrm{P}, \mathrm{TEF}$ ).

Large tree to $20 \mathrm{~m}$ tall. Leaves petiolate, the petioles $0.6-1.5 \mathrm{~cm}$ long; lamina coriaceous, symmetric, elliptic, 6-13×3-6 cm, apex rounded to acute, base cuneate; midrib impressed above, symmetric; flowers not seen. Fruit axillary, solitary; pedicels $3-5 \mathrm{~cm}$ long with two bracteoles at junction with pedicel; bracteoles linear, $2 \mathrm{~mm}$ long; receptacle obconical, 10-12 mm long, four angled; 
tepals ovate, $2.5-3.5 \times 1.2-1.5 \mathrm{~cm}$, rounded at apex, constricted at base to distinct stalk (unguiculate); style persistent, $1.2-1.4 \mathrm{~cm}$ long, 4-fid; disc quadrangular, $1 \mathrm{~cm}$ broad.

Distribution and habitat. - Northern Madagascar, 0-50 m; there are no data on habitat (Fig. 3G).

Additional specimens examined. MADAGASCAR. Known only from the type and one other collection from near the type locality. Antisiranana: Ambanja, 4 Aug 1951, SF4142 (TAN, TEF).

This is the only Madagascan species with fruit comparable in size to those of $F$. mauritiana and $F$. rodriguesiana, but it differs from both in many other characters.

Foetidia vohemarensis Bosser, Adansonia 10: 114. 1988. Type: Madagascar. Antisiranana: Analafiana-Vohêmar, 14 Dec 1955 (fl), Service Forestier du Madagascar, without collector SF15669 (holotype: P; isotype: TEF).

Shrub or tree to $15 \mathrm{~m}$ tall. Leaves shortly petiolate, the petioles 2-4 mm long; lamina coriaceous or chartaceous, obovate, slightly asymmetric, $1.5-6 \times 1-4.5 \mathrm{~cm}$, apex rounded or slightly retuse, base cuneate; midrib plane above. Flowers axillary, solitary; pedicels 2$2.5 \mathrm{~cm}$ long, with 2 bracteoles inserted at junction with receptacle; bracteoles narrowly triangular, 1-1.5 mm long; receptacle obconical, 4 sided, $5 \mathrm{~mm}$ long; tepals oblong, 1.5-1.8× $0.6-0.8 \mathrm{~cm}$, rounded at apex slightly constricted towards base; stamens numerous ca. 70, $13 \mathrm{~mm}$ long; ovary 4 locular with 5 ovules per loculus; style $1 \mathrm{~cm}$ long, 4-fid; disc slightly quadrangular, with a denticulate margin, $0.7-1 \mathrm{~cm}$ broad in fruit. Fruit with persistent tepals $2-2.5 \times$ $0.9-1 \mathrm{~cm}$, chartaceous.

Distribution and habitat.-Confined to Northern Madagascar, in Antisiranana province only, in rainforest up to $1000 \mathrm{~m}$ (Fig. 3H).

Selected specimens examined. MADAGASCAR. Antisiranana: Montagne des Français, $12^{\circ} 22^{\prime} 5^{\prime \prime} \mathrm{S}$, $49^{\circ}$ 21'E, 1000 m, 16 Oct 1954, Capuron SF11320 (P, TEF);
Analafina, $13^{\circ} 26^{\prime} \mathrm{S}, 4^{\circ} 55^{\prime} \mathrm{E}, 11$ Mar 1967, Capuron $S F$ 27504 (K, P, TEF).

\section{Unplaced name}

Foetidia velutina Blume, nom. nud.

\section{Excluded name}

Foetidia ophirensis Knuth=Anneslea fragrans Wall. (Ternstroemiaceae).

\section{Acknowledgments}

I am grateful to the Royal Botanic Gardens, Kew for providing the facilities to carry out this work, to Margaret Tebbs for providing the illustration, to Scott Mori for comments on an earlier draft, and to the herbaria of MO and $\mathrm{P}$ for the loan of material. The maps were drawn by Hannah Stevens, GIS Laboratory, The New York Botanical Garden.

\section{Open Access}

This article is distributed under the terms of the Creative Commons Attribution Noncommercial License which permits any noncommercial use, distribution, and reproduction in any medium, provided the original author(s) and source are credited.

\section{Literature cited}

Bosser, J. 1988. Espècies nouvelles du genre Foetidia (Lecythidaceae) de Madagascar. Adansonia 10: 105119.

Knuth, R. 1939. Barringtoniaceae. In: A. Engler (ed.), Pflanzenreich IV. 219: 62-65. Wilhelm Englemann, Leipzig.

Mori, S. A., C.-H. Tsou, C.-H. Wu, B. Cronholm \& A. Anderberg. 2007. Evolution of Lecythidaceae with an emphasis on the circumscription of Neotropical genera: information from combined ndhF and trnL-F sequence data. American Journal of Botany 94: 289301.

Tsou, C.-H. 1994. The embryology, reproductive morphology, and systematics of Lecythidaceae. Memoirs of the New York Botanical Garden 71: 1-110.

Verdcourt, B. 1985. A new species of Foetidia (Lecythidaceae) from Africa. Kew Bulletin 40: 635636. 


\section{List of exsiccatae}

The numbers in the list correspond to the species of Foetidia below.

1. F. africana B. Verdcourt

2. F. asymmetrica H. Perrier

3. F. capuronii Bosser

4. F. clusioides Baker

5. F. cuneata Bosser

6. F. delphinensis Bosser

7. F. dracaenoides Capuron ex Bosser

8a. F. macrocarpa Bosser var. macrocarpa

8b. F. macrocarpa Bosser var. ambilobensis Bosser

9. F. mauritiana Lam.

10. F. obliqua Blume

11. F. parviflora Capuron ex Bosser

12. F. pterocarpa Bosser

13. F. retusa Blume

14. F. rodriguesiana $\mathrm{F}$. Friedmann

15. F. rubescens Bosser

16. F. sambiranensis Bosser

Andrianantoanina, O. 741 (7)

Aublet, J. B. C. F. s.n. (9)

Balfour, I. B. s.n. (14)

Baron, R. 1394, 2507, 5007, 5011 (10); 5401 (13); 6250 (4)

Bégué, L. H. SF13194 (6)

Bernier, A. C. J. 187, 293 (10)

Betohafara, P. SF15669 (17)

Bijou, F. s.n. (9)

Boivin, B. s.n. (9); s.n. (10)

Bosser, J. 5832 (13); 16537 (2)

Bouton L. s.n. (14)

Cadet, T. 2435 (14); 3710, 4977 (9)

Capuron, R. SF6823, SF7983 (2); SF11236 (11); SF11320 (17); SF11390 (16); SF18065, SF18244 (4); SF18560 (2); SF18705, SF18883bis (8a); SF22093 (5); SF22714 (2); SF23005 (7); SF23852 (5); SF24069 (8a); SF24429, SF24453bis (7); SF24634 (3); SF24740 (15); SF24896 (12); SF25841 (2); SF27504 (17); SF28269 (9); SF28927 (13)
Chapelier, L. A. s.n. (10)

Commerson, P. s.n. 1769 (9)

Decary, M. R. 2851 (13); 6473, 10120 (10)

Debray, C. M. 1514 (13)

Descoings, B. 354 (13)

Floret, J. J. 2001 (10)

Fouchère s.n. 1919 (4)

Friedmann, F. 2578 (9); 3289, 3377 (14)

Gentry, A. H. 11530 (13)

Gereau, R. E. et al. 3315 (10), 3285 (10)

Gerrard, P. 17 (10)

Governor of Mauritius s.n.(9)

Griffith, W. s.n. (9)

Hildebrandt, J. M. 3408 (13)

Humbert, H. 19227bis (7)

Humblot, L. 200 (10)

Leandri, J. et al. 2142 (2)

Lesouef, J.-Y. 3, 32 (9)

Lorence, D. 1181, 2698 (9)

Lowry II, P. P. et al. 3969, 3999 (10)

Mabberley, D. J. 736 (13)

Miller, J. S. et al. 8800(10)

Mwasumbi, L. B. 11607 (1)

Noyes, R. D. et al. 1019 (2)

Perrier de la Bâthie, H. 6527, 6536, 6935 (2)

Pervillé, A. 669a (13)

Rabenantroandro et al. 465 (3)

Rabevohitra, R. 325 (10)

Rahajasoa, G. M. et al. 947 (5)

Raholivelo, L. C. et al. 3 (10)

Rakotomalaza, P. J. et al. 662 (13)

Rakotondrajaona R. et al. 354 (8b)

Randrianaivo, R. et al. 383 (13)

Randriatafika et al. 45 (10)

Ratovoson et al. 746 (4)

Razafimandibison, S. G. 157 (10)

Razakamalala, R. 64 (5); 1488 (10)

Richard, J. M. C. 104 (9); 248, 304 (10); 445 (9); s.n. (10)

Rouxeville, F. 31 (13)

Roxburgh, W. s.n.(9)

Schatz, G. E. et al. 1339, 1795 (10)

Scott Elliot, G. F. 2620 (10) 
Service Forestier du Madagascar SF1848 (13); SF2640 (4); SF3465 (13); SF4142 (16); SF7983 (2); SF7984 (13); SF9739 (6); SF10349 (4); SF13194 (6); SF14108 (13); SF15669 (17); SF 19436 (10); SF19548 (8b); SF25710
(4); SF25811 (2); SF34116, SF 34296 (10); SF34343 (13)

Thouars, L. M. A. du Petit s.n. (10)

Thouvenot, E. 139 (2)

Vaughan, J. H. 651 (10)

Zarucchi, J. L. et al. 7430 (10)

Without Collector s.n.(10); 19426 (10) 